\title{
THE REGULARITY OF THE WAVE EQUATION WITH PARTIAL DIRICHLET CONTROL AND COLOCATED OBSERVATION*
}

\author{
BAO-ZHU GUO ${ }^{\dagger}$ AND XU ZHANG ${ }^{\ddagger}$
}

\begin{abstract}
In this paper we analyze a multidimensional controlled wave equation on a bounded domain, subject to partial Dirichlet control and colocated observation. By means of a partial Fourier transform, it is shown that the system is well-posed and regular in the sense of D. Salamon and G. Weiss. The corresponding feedthrough operator is found to be the identity operator on the input space.
\end{abstract}

Key words. wave equation, transfer function, well-posed and regular system, partial Dirichlet control and colocated observation, partial Fourier transform

AMS subject classifications. 35J05, 93C20, 93C25

DOI. $10.1137 / 040610702$

1. Introduction. A very general class of linear infinite-dimensional systems for which there is a well established theory parallel to that for finite-dimensional systems is the class of well-posed and regular linear systems (see [5]). This generic framework covers many systems governed by partial differential equations with actuators and sensors supported on isolated points, on a subdomain, or on a part of the boundary of the spatial region. There are many papers in this field (e.g., [7], [13], [14], [15], [16], [20], [21], [24], [25], [26], [27], [34], [35], [36], [38], and the references therein). Recently, the regular linear system theory has been generalized to the time-varying case in [22]. We refer to [5] for a nice earlier summary of well-posed system theory.

Well-posedness and regularity are two new crucial concepts introduced in linear infinite-dimensional systems theory under the above-mentioned framework. It is notable that these two concepts are completely different from those one usually uses in partial differential equations. For the reader's convenience, we shall recall their definitions and other related notions in section 2. As remarked in [4], very little is known about the well-posedness or the regularity of controlled infinite-dimensional systems. In [2], the well-posedness of the wave equation with Dirichlet input and colocated output in a two-dimensional (2-D) disk was proved by a direct method. The well-posedness of the same equation on a bounded open domain of $\mathbb{R}^{n}(n \geq 2)$ with a smooth boundary was proved in [1] using microlocal analysis. The well-posedness and regularity of the multidimensional heat equation with both Dirichlet- and Neumanntype boundary control has been established in [3]. To the best of our knowledge, [3] is the first article dealing with the regularity of a multidimensional partial differential equation system, although well-posedness and regularity have been well-established

${ }^{*}$ Received by the editors June 28, 2004; accepted for publication (in revised form) May 28, 2005; published electronically November 14, 2005.

http://www.siam.org/journals/sicon/44-5/61070.html

${ }^{\dagger}$ Corresponding author. Academy of Mathematics and System Sciences, Academia Sinica, Beijing 100080, China, and School of Computational and Applied Mathematics, University of the Witwatersrand, Johannesburg, South Africa (bzguo@iss.ac.cn). This author was supported by the National Natural Science Foundation of China and the National Research Foundation of South Africa.

${ }^{\ddagger}$ School of Mathematics, Sichuan University, Chengdu 610064, China, and Departamento de Matemáticas, Facultad de Ciencias, Universidad Autónoma de Madrid, 28049 Madrid, Spain (xu.zhang@uam.es). This author was supported by the FANEDD of China No. 200119, the NSFC under grant 10371084, the Program for New Century Excellent Talents in University of China, and grant BFM2002-03345 from the Spanish MCYT. 
for many one-dimensional systems (see [11]). The regularity of the wave equation in a 2-D disk with Dirichlet control and colocated observation was first obtained in [12]. However, the same problem for a general bounded domain in $\mathbb{R}^{n}$ has remained open.

The aim of this paper is to give a positive solution to the above-mentioned problem. More precisely, we consider the following multidimensional wave equation with partial Dirichlet control and colocated observation:

$$
\begin{cases}w_{t t}(x, t)-\Delta w(x, t)=0, & x \in \Omega, t>0 \\ w(x, t)=0, & x \in \Gamma_{1}, t>0 \\ w(x, t)=u(x, t), & x \in \Gamma_{0}, t>0 \\ y(x, t)=-\frac{\partial(-\Delta)^{-1} w_{t}(x, t)}{\partial \nu}, & x \in \Gamma_{0}, t>0\end{cases}
$$

Here, $\Omega \subset \mathbb{R}^{n}(n \geq 2)$ is a bounded domain with the smooth boundary $\partial \Omega=\overline{\Gamma_{0}} \cup \overline{\Gamma_{1}}$, both $\Gamma_{0}$ and $\Gamma_{1}$ are disjoint parts of the boundary relatively open in $\partial \Omega$ with int $\left(\Gamma_{0}\right) \neq$ $\emptyset$, and $\nu$ is the unit normal vector of $\Gamma_{0}$ pointing towards the exterior of $\Omega$. In system (1.1), $u$ is the input function (or control) and $y$ is the output function (or output). Put $\mathcal{H}=L^{2}(\Omega) \times H^{-1}(\Omega)$ and $U=L^{2}\left(\Gamma_{0}\right)$. The following result comes from Proposition 2.2 of [1] and Theorem 4.2 of $[19$, p. 46] (see also [17]).

ThEOREM 1.1. Let $T>0,\left(w_{0}, w_{1}\right) \in \mathcal{H}$, and $u \in L^{2}(0, T ; U)$. Then there exists a unique solution $\left(w, w_{t}\right) \in C([0, T] ; \mathcal{H})$ to $(1.1)$ satisfying $w(\cdot, 0)=w_{0}$ and $w_{t}(\cdot, 0)=w_{1}$. Moreover, there exists a constant $C>0$, independent of $\left(w_{0}, w_{1}, u\right)$, such that

$$
\left\|\left(w(\cdot, T), w_{t}(\cdot, T)\right)\right\|_{\mathcal{H}}^{2}+\|y\|_{L^{2}(0, T ; U)}^{2} \leq C\left[\left\|\left(w_{0}, w_{1}\right)\right\|_{\mathcal{H}}^{2}+\|u\|_{L^{2}(0, T ; U)}^{2}\right] .
$$

Theorem 1.1 implies that the system described by (1.1) is well-posed with state space $\mathcal{H}$, input space $U$, and output space $U$ (the precise definition of these concepts will be given in the next section). We mention that Proposition 2.2 of [1] says that there exists a $C^{*}>0$ independent of $u$ such that

$$
\|y\|_{L^{2}(0, T ; U)}^{2} \leq C^{*}\|u\|_{L^{2}(0, T ; U)}^{2} \text { when }\left(w_{0}, w_{1}\right)=0 .
$$

However, as was indicated in [2] and [37], Theorem 1.1 can be derived from here with relative ease.

The main goal of this paper is to show that the system described by (1.1) is regular as well. Our result reads as follows.

TheOrem 1.2. System (1.1) is regular. More precisely, if $w(\cdot, 0)=w_{t}(\cdot, 0)=0$ and $u(x, t) \equiv u(x)$ is a step input with some $u \in U$, then the corresponding output $y$ satisfies

$$
\lim _{\sigma \rightarrow 0} \int_{\Gamma_{0}}\left|\frac{1}{\sigma} \int_{0}^{\sigma} y(x, t) d t-u(x)\right|^{2} d x=0
$$

This result allows us to study dynamic stabilization, optimal control, or other problems for system (1.1) using a theory that is parallel in many ways to the finitedimensional theory; see, e.g., [6]. Also, as we shall explain in section 2, Theorem 1.2 states that system (1.1) has feedthrough operator $\mathbb{D}=I$, where $I$ is the identity operator on $U$.

This paper is organized as follows: In the next section, we introduce the background and the necessary preliminaries about well-posed and regular systems. The proof of Theorem 1.2 is given in section 3 . 
2. Preliminaries. In this section, we shall briefly recall some background about infinite-dimensional well-posed and regular systems (see [5], [27], [30], [31], [32], [33], $[34])$.

Let $X, U$, and $Y$ be three Hilbert spaces. Denote by $\|\cdot\|$ the norm of $X$ (induced by its inner product). In what follows, we choose $X, U$, and $Y$ to be the state, input, and output spaces, respectively, of an infinite-dimensional linear system. This system is described by the equations

$$
\left\{\begin{array}{l}
\dot{x}(t)=\mathbb{A} x(t)+\mathbb{B} u(t), \quad x(0)=x_{0} \in X \\
y(t)=\mathbb{C}_{e} x(t)+\mathbb{D}_{e} u(t)
\end{array}\right.
$$

where the (usually unbounded) operator $\mathbb{A}$ generates a $C_{0}$-semigroup $\mathbb{T}(\cdot)$ on $X, \mathbb{B}$ is a control operator from $U$ to $X, \mathbb{C}_{e}$ is an observation operator from $X$ to $Y$, and $\mathbb{D}_{e}$ is a bounded operator from $U$ to $Y$. In $(2.1), u(t) \in U, x(t) \in X$, and $y(t) \in Y$ are called the input, the state, and the output, respectively. The input function $u(\cdot)$ is assumed to be in the space $L_{\text {loc }}^{2}(0, \infty ; U)$, but the representation (2.1) is valid only if $u \in H_{l o c}^{1}(0, \infty ; U)$ and $\mathbb{A} x(0)+\mathbb{B} u(0) \in X$ (see [28] for details). For the case that both $\mathbb{B}$ and $\mathbb{C}_{e}$ are bounded, a nice theory for system (2.1) has been summarized in the book [9]. The framework of well-posed system theory is, however, mainly concerned with the case where neither $\mathbb{B}$ nor $\mathbb{C}_{e}$ is bounded.

Let us recall some basic notation. The Hilbert space $X_{-1}$ is defined as the completion of $X$ with respect to the norm

$$
\|x\|_{-1}=\left\|(\beta-\mathbb{A})^{-1} x\right\| \quad \forall x \in X,
$$

and the space $X_{1}$ is the space $D(\mathbb{A})$ with the norm

$$
\|x\|_{1}=\|(\beta-\mathbb{A}) x\| \quad \forall x \in D(\mathbb{A}),
$$

where $\beta \in \rho(\mathbb{A})$, the resolvent set of $\mathbb{A}$. It is easy to verify that both $X_{-1}$ and $X_{1}$ are independent of the choice of $\beta$. It was shown in [30] that $X_{-1}=D\left(\mathbb{A}^{*}\right)^{\prime}$, the dual space of $D\left(\mathbb{A}^{*}\right)$ with respect to the pivot $X$. Identifying $X$ with its dual space, we have the following continuous, dense inclusions:

$$
X_{1} \hookrightarrow X \hookrightarrow X_{-1}
$$

Definition 2.1. System (2.1) is said to be well-posed if the following hold:

(a) $\mathbb{A}$ generates a $C_{0}$-semigroup $\mathbb{T}(\cdot)$ on $X$.

(b) $\mathbb{B} \in \mathcal{L}\left(U, X_{-1}\right)$ is an admissible control operator for $\mathbb{T}(\cdot)$, i.e., for some (and hence for any) $t>0$ there exists $C_{t}>0$ such that

$$
\left\|\int_{0}^{t} \mathbb{T}(t-\tau) \mathbb{B} u(\tau) d \tau\right\|^{2} \leq C_{t} \int_{0}^{t}\|u(t)\|_{U}^{2} d t \quad \forall u \in L^{2}(0, t ; U) .
$$

(c) The domain $D\left(\mathbb{C}_{e}\right) \supset D(\mathbb{A})$. If we denote by $\mathbb{C}$ the restriction of $\mathbb{C}_{e}$ to $D(\mathbb{A})$, then $\mathbb{C} \in \mathcal{L}\left(X_{1}, Y\right)$ is an admissible observation operator for $\mathbb{T}(\cdot)$, which means that for some (and hence for any) $t>0$, there exists $C_{t}^{\prime}>0$ such that

$$
\int_{0}^{t}\|\mathbb{C T}(\cdot) x\|_{Y}^{2} d t \leq C_{t}^{\prime}\|x\|^{2} \quad \forall x \in D(\mathbb{A}) .
$$


(d) The input-output map is bounded; i.e., for some (and hence for any) $t>0$, there exists $C_{t}^{\prime \prime}>0$ such that

$$
\int_{0}^{t}\|y(t)\|_{Y}^{2} d t \leq C_{t}^{\prime \prime} \int_{0}^{t}\|u(t)\|_{U}^{2} d t \quad \forall u \in L^{2}(0, t ; U) \text { when } x_{0}=0 .
$$

It should be noted that the definition above is not the standard one given by [5] or [8], but it is equivalent to Weiss's definition (see [16], [23], [27]). From [31], $\mathbb{B}$ is admissible for $\mathbb{T}(\cdot)$ if and only if the adjoint operator $\mathbb{B}^{*}$ is admissible for $\mathbb{T}^{*}(\cdot)$, the adjoint $C_{0}$-semigroup of $\mathbb{T}(t)$.

Roughly speaking, a well-posed system is a system for which both the state and output depend continuously on the initial state and input function of the system.

If system (2.1) is well-posed, then the weak solution of (2.1) can be represented as (see [5], [28])

$$
\left\{\begin{aligned}
x(t) & =\mathbb{T}(t) x_{0}+\int_{0}^{t} \mathbb{T}(t-\tau) \mathbb{B} u(\tau) d \tau \in C([0, \infty) ; X) \\
& \forall x_{0} \in X, u \in L_{l o c}^{2}(0, \infty ; U), \\
y(t) & =\mathbb{C}_{\Lambda}\left[x(t)-(\lambda-\mathbb{A})^{-1} \mathbb{B} u(t)\right]+\mathbb{H}(\lambda) u(t) \in L_{l o c}^{2}(0, \infty ; Y) \\
& \forall u \in L_{l o c}^{2}(0, \infty ; U),
\end{aligned}\right.
$$

where $\mathbb{C}_{\Lambda} x=\lim _{\lambda \rightarrow+\infty} \mathbb{C} \lambda(\lambda-\mathbb{A})^{-1} x$ for all $x \in D\left(\mathbb{C}_{\Lambda}\right)$ is by definition the $\Lambda$ extension of $\mathbb{C}$, where $D\left(\mathbb{C}_{\Lambda}\right)$ is the subspace of $X$ for which the associated limit exists (see [5]). $\mathbb{H}(\lambda)$ is called the transfer function which is defined in some right-half planes and is an analytic $\mathcal{L}(U, Y)$-valued function. It can be shown that if $\hat{u}(\lambda)$ exists, then

$$
\hat{y}(\lambda)=\mathbb{H}(\lambda) \hat{u}(\lambda) \quad \text { when } x_{0}=0,
$$

where^ denotes the Laplace transform. In terms of the operators from (2.1), we have (see $[28])$

$$
\mathbb{H}(\lambda)=\mathbb{C}_{e}(\lambda-\mathbb{A})^{-1} \mathbb{B}+\mathbb{D}_{e} .
$$

The transfer function $\mathbb{H}(\lambda)$ can be determined by the triple of operators $(\mathbb{A}, \mathbb{B}, \mathbb{C})$ up to an additive constant bounded operator in the following way (see [8]):

$$
\frac{\mathbb{H}(\lambda)-\mathbb{H}(\beta)}{\lambda-\beta}=-\mathbb{C}(\lambda-\mathbb{A})^{-1}(\beta-\mathbb{A})^{-1} \mathbb{B} \quad \forall \lambda, \beta \in \mathcal{C}_{\rho}^{+}, \lambda \neq \beta,
$$

where $\mathcal{C}_{\rho}^{+}=\{\lambda \in \mathcal{C} \mid \operatorname{Re} \lambda>\rho\}$ for some $\rho>0$ and $\mathcal{C}$ stands for the complex plane. Using the transfer function, the boundedness of the input-output map described in condition (d) of Definition 2.1 can be expressed as the boundedness of the transfer function on an open right complex half plane (see [8], [11], [16])

$$
\sup _{\operatorname{Re} \lambda \geq \alpha>\rho}\|\mathbb{H}(\lambda)\|_{\mathcal{L}(U, Y)}<\infty
$$

for some $\alpha \in \mathbb{R}$.

The paper [32] introduced an important subclass of well-posed systems, the socalled regular systems, for which the representation (2.2) becomes much simpler. 
Definition 2.2. System (2.1) is said to be regular if it is well-posed and there exists an operator $\mathbb{D} \in \mathcal{L}(U, Y)$ such that, for $x_{0}=0$ and $u(t) \equiv u \in U$, the output $y$ of (2.1) satisfies

$$
\lim _{t \rightarrow 0} \frac{1}{t} \int_{0}^{t} y(\tau) d \tau=\mathbb{D} u
$$

in the strong topology of $Y$. The above $\mathbb{D}$ and property (2.6) are called the feedthrough operator and the regularity of system (2.1), respectively.

It was shown in [34] that, in the frequency domain, (2.6) is equivalent to

$$
\lim _{\lambda \in \mathbb{R}, \lambda \rightarrow+\infty} \mathbb{H}(\lambda) u=\mathbb{D} u \quad \forall u \in U .
$$

If a well-posed system is regular, then (2.2) can be written as

$$
\left\{\begin{aligned}
x(t) & =\mathbb{T}(t) x_{0}+\int_{0}^{t} \mathbb{T}(t-\tau) \mathbb{B} u(\tau) d \tau \in C([0, \infty) ; X), \\
& x_{0} \in X, u \in L_{l o c}^{2}(0, \infty ; U), \\
y(t) & =\mathbb{C}_{\Lambda} x(t)+\mathbb{D} u(t) \in L_{l o c}^{2}(0, \infty ; Y), u \in L_{l o c}^{2}(0, \infty ; U) .
\end{aligned}\right.
$$

In this case, the transfer function is uniquely determined by the quadruple of operators $(\mathbb{A}, \mathbb{B}, \mathbb{C}, \mathbb{D})$ and can be represented as

$$
\mathbb{H}(\lambda)=\mathbb{D}+\mathbb{C}_{\Lambda}(\lambda-\mathbb{A})^{-1} \mathbb{B} .
$$

It is seen that the representations (2.8) and (2.9) resemble that for finite-dimensional systems.

Roughly speaking, a well-posed regular system is like a linear finite-dimensional system among the infinite-dimensional systems but with the feature of allowing both control and observation operators to be unbounded in some sense. Unlike stability, controllability, observability, etc., which have finite-dimensional counterparts, regularity is an important but new concept in linear infinite-dimensional systems under the elegant framework of well-posed linear systems theory.

Now let us introduce a special class of well-posed systems: the colocated secondorder linear systems. It is well known that "passivity," which was introduced in connection with circuit theory in the 1950s (see [10]), is a very important concept in control system design. It means that the increase of energy stored in the system does not exceed the energy that enters from the external world. For such a system, the transfer function is positive real, and negative output feedback produces a dissipative system, which is stable in the sense of Lyapunov. For a long time, it has been known by engineers that a partial differential equation describing a mechanical system, like a flexible structure in which the power flow into the system is the scalar product $\langle u, y\rangle$ (e.g., when $u$ is force and $y$ is velocity), leads to a positive-real system (2.1) in which $U=Y$ and $\mathbb{A}^{*}+\mathbb{A} \leq 0, \mathbb{C}=\mathbb{B}^{*}$ if actuators and sensors are designed in a "colocated" fashion. The particular case $\mathbb{A}+\mathbb{A}^{*}=0$ corresponds to energy preserving systems. This means that the measurement and control action are made dual in some sense. In [11] and [35], an abstract setting of a second-order passive system of the following type was studied. The state space is $X=D\left(\mathbb{A}_{0}^{1 / 2}\right) \times H$, and the input and output spaces are the same $U=Y$ (see also [2], [37]):

$$
\left\{\begin{array}{l}
\ddot{x}(t)+\mathbb{A}_{0} x(t)=\mathbb{B}_{0} u(t) \\
y(t)=\mathbb{B}_{0}^{*} \dot{x}(t)
\end{array}\right.
$$


where

(i) $\mathbb{A}_{0}: D\left(\mathbb{A}_{0}\right)(\subset H) \rightarrow H$ is an unbounded positive self-adjoint operator in the Hilbert space $H$;

(ii) $\mathbb{B}_{0} \in \mathcal{L}\left(U,\left(D\left(\mathbb{A}_{0}^{1 / 2}\right)\right)^{\prime}\right)$;

(iii) $\mathbb{B}_{0}^{*} \in \mathcal{L}\left(D\left(\mathbb{A}_{0}^{1 / 2}\right), U\right)$ is defined as

$$
\left(\mathbb{B}_{0}^{*} x, u\right)_{U}=\left\langle x, \mathbb{B}_{0} u\right\rangle_{D\left(\mathbb{A}_{0}^{1 / 2}\right) \times\left(D\left(\mathbb{A}_{0}^{1 / 2}\right)\right)^{\prime}} \quad \forall x \in D\left(\mathbb{A}_{0}^{1 / 2}\right) ;
$$

(iv) an extension $\tilde{\mathbb{A}}_{0} \in \mathcal{L}\left(D\left(\mathbb{A}_{0}^{1 / 2}\right),\left(D\left(\mathbb{A}_{0}^{1 / 2}\right)\right)^{\prime}\right)$ of $\mathbb{A}_{0}$ is defined by

$$
\left\langle\tilde{\mathbb{A}}_{0} x, z\right\rangle_{\left(D\left(\mathbb{A}_{0}^{1 / 2}\right)\right)^{\prime} \times D\left(\mathbb{A}_{0}^{1 / 2}\right)}=\left(\mathbb{A}_{0}^{1 / 2} x, \mathbb{A}_{0}^{1 / 2} z\right)_{H} \quad \forall x, z \in D\left(\mathbb{A}_{0}^{1 / 2}\right) .
$$

It was found in [11] that if system (2.10) is well-posed, its transfer function is uniquely determined by the pair $\left(\mathbb{A}_{0}, \mathbb{B}_{0}\right)$ :

$$
H(\lambda)=\lambda \mathbb{B}_{0}^{*}\left(\lambda^{2}+\tilde{\mathbb{A}}_{0}\right)^{-1} \mathbb{B}_{0}
$$

Actually, it was indicated in [2] and [37] that, for this system, the boundedness of the transfer function on some open right half complex plane implies automatically the admissibility of $\left[\begin{array}{c}0 \\ \mathbb{B}_{0}\end{array}\right]$ for the associated semigroup generated by $\mathbb{A}=\left[\begin{array}{cc}0 & I \\ -\mathbb{A}_{0} & 0\end{array}\right]$. This system is closely related (via feedback) to the example in [29].

To end this section, we return to our wave equation (1.1) with control $u \in$ $L_{l o c}^{2}(0, \infty ; U), U=L^{2}\left(\Gamma_{0}\right)$. We formulate our problem in the framework of $(2.10)$, although it is already available in the literature (see, e.g., [1]).

Let $H=H^{-1}(\Omega)$ be the dual space of the usual Sobolev space $H_{0}^{1}(\Omega)$ (with respect to the pivot space $\left.L^{2}(\Omega)\right)$. Let $A_{0}$ be the positive self-adjoint operator in $H$ induced by the bilinear form $a(\cdot, \cdot)$ defined by

$$
\left\langle A_{0} f, g\right\rangle_{H^{-1}(\Omega) \times H_{0}^{1}(\Omega)}=a(f, g)=\int_{\Omega} \nabla f(x) \overline{\nabla g(x)} d x \quad \forall f, g \in H_{0}^{1}(\Omega) .
$$

By means of the Lax-Milgram theorem, $A_{0}$ is a canonical isomorphism from $D\left(A_{0}\right)=$ $H_{0}^{1}(\Omega)$ to $H$. If we introduce the Laplacian $-\Delta: H^{2}(\Omega) \cap H_{0}^{1}(\Omega) \rightarrow L^{2}(\Omega)$, then it is easy to show that $A_{0} f=-\Delta f$ for $f \in H^{2}(\Omega) \cap H_{0}^{1}(\Omega)$ and that $A_{0}^{-1} g=(-\Delta)^{-1} g$ for any $g \in L^{2}(\Omega)$. Hence, $A_{0}$ is an extension of usual Laplacian to the space $H_{0}^{1}(\Omega)$.

It is well known that $D\left(A_{0}^{1 / 2}\right)=L^{2}(\Omega)$. Define the Dirichlet map

$$
\Upsilon \in \mathcal{L}\left(L^{2}\left(\Gamma_{0}\right), L^{2}(\Omega)\right),
$$

i.e., $\Upsilon u=v$ by

$$
\left\{\begin{array}{l}
\Delta v=0 \quad \text { in } \Omega \\
\left.v\right|_{\Gamma_{1}}=0,\left.\quad v\right|_{\Gamma_{0}}=u
\end{array}\right.
$$

Using the Dirichlet map, we can rewrite the first three equations in (1.1) as

$$
\ddot{w}+A_{0}(w-\Upsilon u)=0
$$

We identify $H$ with its dual $H^{\prime}$. Then the following relations hold:

$$
D\left(A_{0}^{1 / 2}\right) \hookrightarrow H \hookrightarrow\left(D\left(A_{0}^{1 / 2}\right)\right)^{\prime} .
$$


An extension $\tilde{A}_{0} \in \mathcal{L}\left(D\left(A_{0}^{1 / 2}\right),\left(D\left(A_{0}^{1 / 2}\right)\right)^{\prime}\right)$ of $A_{0}$ is defined by

$$
\left\langle\tilde{A}_{0} f, g\right\rangle_{\left(D\left(A_{0}^{1 / 2}\right)\right)^{\prime} \times D\left(A_{0}^{1 / 2}\right)}=\left(A_{0}^{1 / 2} f, A_{0}^{1 / 2} g\right)_{H} \quad \forall f, g \in D\left(A_{0}^{1 / 2}\right) .
$$

Hence, (2.14) can be rewritten in $\mathcal{H}_{-1}$ as

$$
\ddot{w}+\tilde{A}_{0} w=B_{0} u,
$$

where $B_{0} \subset \mathcal{L}\left(U,\left(D\left(A_{0}^{1 / 2}\right)\right)^{\prime}\right)$ is given by

$$
B_{0} u=\tilde{A}_{0} \Upsilon u \quad \forall u \in U .
$$

Define $B_{0}^{*} \in \mathcal{L}\left(D\left(A_{0}^{1 / 2}\right), U\right)$ by

$$
\left(B_{0}^{*} f, u\right)_{U}=\left\langle f, B_{0} u\right\rangle_{D\left(A_{0}^{1 / 2}\right) \times\left(D\left(A_{0}^{1 / 2}\right)\right)^{\prime}} \quad \forall f \in D\left(A_{0}^{1 / 2}\right) .
$$

Then for any $f \in D\left(A_{0}^{1 / 2}\right)$ and $u \in C_{0}^{\infty}\left(\Gamma_{0}\right)$, we have

$$
\begin{aligned}
& \left\langle f, B_{0} u\right\rangle_{D\left(A_{0}^{1 / 2}\right) \times\left(D\left(A_{0}^{1 / 2}\right)\right)^{\prime}}=\left\langle\tilde{A}_{0} f, \tilde{A}_{0}^{-1} B_{0} u\right\rangle_{D\left(A_{0}^{1 / 2}\right) \times\left(D\left(A_{0}^{1 / 2}\right)\right)^{\prime}} \\
& =\left(A_{0}^{1 / 2} f, A_{0}^{1 / 2} \tilde{A}_{0}^{-1} B_{0} u\right)_{H}=\left(A_{0}^{-1} A_{0}^{1 / 2} f, A_{0}^{-1} A_{0}^{1 / 2} \tilde{A}_{0}^{-1} B_{0} u\right)_{H_{0}^{1}(\Omega)} \\
& =\left(A_{0}^{-1 / 2} f, A_{0}^{-1 / 2} \Upsilon u\right)_{H_{0}^{1}(\Omega)}=(f, \Upsilon u)_{L^{2}(\Omega)} \\
& =\left(A_{0} A_{0}^{-1} f, \Upsilon u\right)_{L^{2}(\Omega)}=-\left(\frac{\partial(-\Delta)^{-1} f}{\partial \nu}, u\right)_{U} .
\end{aligned}
$$

In the last step, we used the fact that

$$
\int_{\Omega} \nabla v \nabla \phi=0 \quad \forall \phi \in H_{0}^{1}(\Omega)
$$

holds for any classical solution $v$ of (2.13). Since $C_{0}^{\infty}\left(\Gamma_{0}\right)$ is dense in $L^{2}\left(\Gamma_{0}\right)$, we obtain

$$
B_{0}^{*}=-\left.\frac{\partial(-\Delta)^{-1}}{\partial \nu}\right|_{\Gamma_{0}} .
$$

Now, we have formulated system (1.1) into an abstract form of the second-order system (2.10) in the state space $\mathcal{H}$ :

$$
\left\{\begin{array}{l}
\ddot{w}(t)+\tilde{A}_{0} w(t)=B_{0} u(t), \\
y(t)=B_{0}^{*} \dot{w}
\end{array}\right.
$$

where $B_{0}$ and $B_{0}^{*}$ are defined by (2.17) and (2.18), respectively.

The main contribution of this paper is to show that system (2.19) is regular with feedthrough operator $\mathbb{D}=I$.

3. Proof of Theorem 1.2. From (2.19), we see that system (1.1) is in the framework of form (2.10) discussed in section 2. Since system (1.1) is well-posed, it follows from (2.11) that the transfer function of system (1.1) is

$$
H(\lambda)=\lambda B_{0}^{*}\left(\lambda^{2}+\tilde{A}_{0}\right)^{-1} B_{0},
$$


where $\tilde{A}_{0}, B_{0}$, and $B_{0}^{*}$ are given by (2.15), (2.17), and (2.18), respectively. Moreover, from the well-posedness and (2.5), it follows that there exists a positive number $\alpha>0$ such that

$$
\sup _{\operatorname{Re} \lambda \geq \alpha}\|H(\lambda)\|_{\mathcal{L}(U)}=M<\infty .
$$

To begin, we show the following proposition.

Proposition 3.1. Theorem 1.2 is valid if for any $u \in C_{0}^{\infty}\left(\Gamma_{0}\right)$ the solution $u_{\varepsilon}$ to the equation

$$
\begin{cases}u_{\varepsilon}(x)-\varepsilon^{2} \Delta u_{\varepsilon}(x)=0, & x \in \Omega, \\ u_{\varepsilon}(x)=0, & x \in \Gamma_{1}, \\ u_{\varepsilon}(x)=u(x), & x \in \Gamma_{0}\end{cases}
$$

satisfies

$$
\lim _{\varepsilon \rightarrow 0} \int_{\Gamma_{0}}\left|\varepsilon \frac{\partial u_{\varepsilon}(x)}{\partial \nu}-u(x)\right|^{2} d x=0
$$

where $\varepsilon$ are real and positive numbers.

Proof. In light of the equivalence between (2.6) and (2.7), in order to prove Theorem 1.2 we need only to show that

$$
\lim _{\lambda \in \mathbb{R}, \lambda \rightarrow+\infty} H(\lambda) u=u
$$

for any $u \in L^{2}\left(\Gamma_{0}\right)=U$ in the strong topology of $U$, where $H(\lambda)$ is given by (3.1). We claim that in order to show (3.5), it suffices to show that (3.5) is satisfied for all $u \in C_{0}^{\infty}\left(\Gamma_{0}\right)$. Indeed, for any $u \in U$ and any given $\delta>0$, since $C_{0}^{\infty}\left(\Gamma_{0}\right)$ is dense in $L^{2}\left(\Gamma_{0}\right)$, if (3.5) is valid for $u \in C_{0}^{\infty}\left(\Gamma_{0}\right)$, then one can find $u_{0} \in C_{0}^{\infty}\left(\Gamma_{0}\right)$ and the real number $\beta>\alpha$ such that

$$
\left\|u_{0}-u\right\|_{U}<\min \left\{\frac{\delta}{3 M}, \frac{\delta}{3}\right\}, \quad \sup _{\lambda \in \mathbb{R}, \lambda>\beta}\left\|H(\lambda) u_{0}-u_{0}\right\|_{U}<\frac{\delta}{3},
$$

where $M$ and $\alpha$ are given in (3.2). Therefore,

$$
\sup _{\lambda \in \mathbb{R}, \lambda>\beta}\|H(\lambda) u-u\|_{U}=\sup _{\lambda \in \mathbb{R}, \lambda>\beta}\left\|H(\lambda) u_{0}-u_{0}+H(\lambda)\left(u-u_{0}\right)-u+u_{0}\right\|_{U}<\delta .
$$

This shows that (3.5) is valid for any $u \in U$.

Now assume that $u \in C_{0}^{\infty}\left(\Gamma_{0}\right)$, and put

$$
w_{\lambda}(x)=\left(\left(\lambda^{2}+\tilde{A}_{0}\right)^{-1} B_{0} u\right)(x) .
$$

Then $w_{\lambda}$ satisfies

$$
\begin{cases}\lambda^{2} w_{\lambda}(x)-\Delta w_{\lambda}(x)=0, & x \in \Omega, \\ w_{\lambda}(x)=0, & x \in \Gamma_{1}, \\ w_{\lambda}(x)=u(x), & x \in \Gamma_{0},\end{cases}
$$

and

$$
(H(\lambda) u)(x)=-\lambda \frac{\partial\left((-\Delta)^{-1} w_{\lambda}\right)(x)}{\partial \nu} \quad \forall x \in \Gamma_{0} .
$$


Since $u \in C_{0}^{\infty}\left(\Gamma_{0}\right)$, there exists a unique classical solution to (3.6). Take a function $v \in H^{2}(\Omega)$ such that

$$
\begin{cases}\Delta v(x)=0, & x \in \Omega, \\ v(x)=0, & x \in \Gamma_{1} \\ v(x)=u(x), & x \in \Gamma_{0}\end{cases}
$$

Then (3.6) can be written as

$$
\left\{\begin{array}{l}
\lambda^{2} w_{\lambda}(x)-\Delta\left(w_{\lambda}(x)-v(x)\right)=0, \quad x \in \Omega \\
\left.\left(w_{\lambda}-v\right)\right|_{\partial \Omega}=0
\end{array}\right.
$$

or equivalently

$$
-\lambda^{2}\left((-\Delta)^{-1} w_{\lambda}\right)(x)=w_{\lambda}(x)-v(x)
$$

Hence (3.7) becomes

$$
(H(\lambda) u)(x)=\frac{1}{\lambda} \frac{\partial w_{\lambda}(x)}{\partial \nu}-\frac{1}{\lambda} \frac{\partial v(x)}{\partial \nu}
$$

Letting $u_{\varepsilon}(x)=w_{\lambda}(x)$ with $\varepsilon=\lambda^{-1}$ and noting that $\frac{\partial v(x)}{\partial \nu}$ is independent of $\lambda$, we conclude the required result.

The rest of this section is devoted to proving that the solution $u_{\varepsilon}$ of (3.3) with $u \in C_{0}^{\infty}\left(\Gamma_{0}\right)$ satisfies (3.4). We shall go a little bit further. Indeed, we will show that there exists a constant $C>0$ such that for all $\varepsilon \in(0,1)$, any solution $u_{\varepsilon} \in H^{2}(\Omega)$ of

$$
\left(\varepsilon^{2} \Delta-1\right) u_{\varepsilon}(x)=0, \quad x \in \Omega,
$$

satisfies the following inequality:

$$
\left\|\varepsilon \frac{\partial u_{\varepsilon}}{\partial \nu}-u_{\varepsilon}\right\|_{L^{2}(\partial \Omega)}^{2} \leq C \varepsilon\left\|u_{\varepsilon}\right\|_{H^{3 / 2}(\partial \Omega)}^{2} .
$$

This will be performed by estimating the Dirichlet-Neumann map by means of easy Fourier analysis tools after applying a diffeomorphism to reduce locally our geometry to the half-space. Notice that the Dirichlet-Neumann map for the Laplacian in a manifold was more precisely computed in [18] by using symbolic calculus of pseudodifferential operators.

Proof of Theorem 1.2. By Proposition 3.1, we need only to show that the solution $u_{\varepsilon}$ of (3.3) with $u \in C_{0}^{\infty}\left(\Gamma_{0}\right)$ satisfies (3.4). We assume $0<\varepsilon<1$ throughout the proof.

For any $x_{0} \in \partial \Omega$, suppose without loss of generality that in an open neighborhood $V_{x_{0}} \subset \mathbb{R}^{n}$ of $x_{0}$,

$$
V_{x_{0}} \cap \Omega=\left\{\left(x^{\prime}, x_{n}\right)=\left(x_{1}, x_{2}, \ldots, x_{n-1}, x_{n}\right) \in V_{x_{0}}, x_{n}-\phi\left(x^{\prime}\right)>0\right\}
$$

for some $\phi \in C^{3}\left(\mathbb{R}^{n-1}\right)$. Then the unit outward normal vector to $V_{x_{0}} \cap \partial \Omega$ at $\left(x^{\prime}, \phi\left(x^{\prime}\right)\right)$ is defined by

$$
\nu\left(x^{\prime}\right)=\frac{\left(\partial_{x_{1}} \phi\left(x^{\prime}\right), \ldots, \partial_{x_{n-1}} \phi\left(x^{\prime}\right),-1\right)}{\sqrt{1+\left|\nabla \phi\left(x^{\prime}\right)\right|^{2}}} .
$$


Let us use the geodesic normal coordinates as follows. Let

$$
(h, s)=\left(h_{1}, h_{2}, \ldots, h_{n-1}, s\right) \in \mathbb{R}^{n} .
$$

We introduce a diffeomorphism by

$$
\Psi(h, s)=(h, \phi(h))-s \nu(h)
$$

such that

(i) $\Psi^{-1}\left(\Omega_{x_{0}}\right)=B_{r}=\left\{(h, s) \in \mathbb{R}^{n},|(h, s)|<r\right\}$;

(ii) $\Psi^{-1}\left(\Omega_{x_{0}} \cap \Omega\right)=B_{r}^{+}=\left\{(h, s) \in B_{r}, s>0\right\}$;

(iii) $\Psi^{-1}\left(\Omega_{x_{0}} \cap \partial \Omega\right)=\left\{(h, s) \in B_{r}, s=0\right\}=\{|h|<r\} \times\{0\}$

for some $r>0$ and an open neighborhood $\Omega_{x_{0}}\left(\subset V_{x_{0}}\right)$ of $x_{0}$, where $|\cdot|$ denotes the Euclidean norm. Using the diffeomorphism $\Psi: B_{r} \rightarrow \Omega_{x_{0}}$, the normal derivative on the boundary becomes

$$
\frac{\partial}{\partial \nu}=-\partial_{s}
$$

and the operator in the first equation of (3.3) can be written in the form

$$
\Delta-\frac{1}{\varepsilon^{2}}=\partial_{s}^{2}+P\left(h, s,-i \partial_{h}\right)+\ell(h, s) \partial_{s}-\frac{1}{\varepsilon^{2}},
$$

where $\partial_{h}=\left(\partial_{h_{1}}, \ldots, \partial_{h_{n-1}}\right), \ell$ is a continuous function, and $P$ is a second-order elliptic differential operator in the $h$ variables only.

The proof is now divided into three steps.

Step 1. Flattening and localization. We first flatten the local domain $\Omega_{x_{0}} \cap \Omega$ with the above diffeomorphism $\Psi$ and set

$$
\tilde{u}_{\varepsilon}(h, s)=u_{\varepsilon}(\Psi(h, s)), \quad \tilde{u}(h)=u_{\varepsilon}(\Psi(h, 0)) .
$$

Then $\tilde{u}_{\varepsilon}$ satisfies

$$
\left\{\begin{array}{l}
\partial_{s}^{2} \tilde{u}_{\varepsilon}(h, s)+\sum_{i, j=1}^{n-1} a_{i j}(h, s) \partial_{h_{i}} \partial_{h_{j}} \tilde{u}_{\varepsilon}(h, s)+Q \tilde{u}_{\varepsilon}(h, s)-\frac{1}{\varepsilon^{2}} \tilde{u}_{\varepsilon}(h, s)=0, \\
\tilde{u}_{\varepsilon}(h, 0)=\tilde{u}(h), \quad|h|<r,
\end{array}\right.
$$

where $Q$ is a linear differential operator of order 1 with continuous coefficients in $B_{r}$ and $\left(a_{i j}\right)_{1 \leq i, j \leq n-1}$ is a strictly positive definite symmetric matrix of continuous functions of $(h, s)$ in $B_{r}$. Assume that $\lambda_{0}>0$ is a constant such that

$$
\sum_{i, j=1}^{n-1} a_{i j}(h, s) \xi_{i} \xi_{j} \geq \lambda_{0}|\xi|^{2} \quad \forall \xi=\left(\xi_{1}, \xi_{2}, \ldots, \xi_{n-1}\right) \in \mathbb{R}^{n-1}, \quad(h, s) \in B_{r} .
$$

Let $\mu_{0}>0$ be such that $\mu_{0}<\frac{\lambda_{0}}{(n-1)^{2}}$. Since $a_{i j}$ is continuous in $B_{r}$, one can find a scalar $\rho \in(0, r)$ such that

$$
\left|a_{i j}(h, s)-a_{i j}(0,0)\right| \leq \mu_{0} \quad \forall i, j=1,2, \ldots, n-1, \quad(h, s) \in B_{\rho}^{+} .
$$


Second, we introduce a cutoff function $\varphi=\varphi(h, s) \in C_{0}^{\infty}\left(B_{\rho}\right)$ such that $0 \leq \varphi \leq 1$ and $\varphi=1$ in $B_{\rho / 2}$. Set, for all $(h, s) \in \mathbb{R}^{n-1} \times \mathbb{R}^{+}$,

$$
\chi_{\varepsilon}(h, s)=\varphi(h, s) \tilde{u}_{\varepsilon}(h, s), \quad f(h)=\varphi(h, 0) \tilde{u}(h) .
$$

Then one can check that $\chi_{\varepsilon} \in H^{2}\left(\mathbb{R}^{n-1} \times \mathbb{R}^{+}\right)$and $\chi_{\varepsilon}(h, s)=0$ in $\mathbb{R}^{n-1} \times\{s \geq \rho\}$. By (3.12), $\chi_{\varepsilon}$ satisfies

$$
\left\{\begin{array}{c}
\partial_{s}^{2} \chi_{\varepsilon}(h, s)+\sum_{i, j=1}^{n-1} a_{i j}(0,0) \partial_{h_{i}} \partial_{h_{j}} \chi_{\varepsilon}(h, s)-\frac{1}{\varepsilon^{2}} \chi_{\varepsilon}(h, s) \\
=G \chi_{\varepsilon}(h, s)+L \tilde{u}_{\varepsilon}(h, s), \quad(h, s) \in \mathbb{R}^{n-1} \times \mathbb{R}^{+}, \\
\chi_{\varepsilon}(h, 0)=f(h), \quad h \in \mathbb{R}^{n-1},
\end{array}\right.
$$

where

$$
\left\{\begin{aligned}
G \chi_{\varepsilon}(h, s)= & \sum_{i, j=1}^{n-1}\left[a_{i j}(0,0)-a_{i j}(h, s)\right] \partial_{h_{i}} \partial_{h_{j}} \chi_{\varepsilon}(h, s), \\
L \tilde{u}_{\varepsilon}(h, s)= & -\varphi(h, s) Q \tilde{u}_{\varepsilon}(h, s)+\left[\partial_{s}^{2}, \varphi\right] \tilde{u}_{\varepsilon}(h, s) \\
& +\sum_{i, j=1}^{n-1} a_{i j}(h, s)\left[\partial_{h_{i}} \partial_{h_{j}}, \varphi\right] \tilde{u}_{\varepsilon}(h, s)
\end{aligned}\right.
$$

with

$$
\left[\partial_{s}^{2}, \varphi\right] \tilde{u}_{\varepsilon}=2 \partial_{s} \varphi \partial_{s} \tilde{u}_{\varepsilon}+\partial_{s}^{2} \varphi \tilde{u}_{\varepsilon}, \quad\left[\partial_{h_{i}} \partial_{h_{j}}, \varphi\right] \tilde{u}_{\varepsilon}=\partial_{h_{i}} \varphi \partial_{h_{j}} \tilde{u}_{\varepsilon}+\partial_{h_{j}} \varphi \partial_{h_{i}} \tilde{u}_{\varepsilon}+\partial_{h_{i}} \partial_{h_{j}} \varphi \tilde{u}_{\varepsilon} .
$$

Clearly, $G$ and $L$ are two linear differential operators of order 2 and order 1, respectively.

Step 2. Partial Fourier transform. Fix $s$, for any $\chi(\cdot, s) \in L^{2}\left(\mathbb{R}^{n-1}\right)$. From now on, we denote by $\widehat{\chi}(\xi, s)$ the partial Fourier transform of $\chi(h, s)$ with respect to $h$, i.e.,

$$
\widehat{\chi}(\xi, s)=\int_{\mathbb{R}^{n-1}} \chi(h, s) e^{-i\langle h, \xi\rangle} d h
$$

Applying the above partial Fourier transform to system (3.16), it becomes

$$
\left\{\begin{aligned}
\partial_{s}^{2} \widehat{\chi_{\varepsilon}}(\xi, s)-\frac{1}{\varepsilon^{2}}\left(\varepsilon^{2} \xi^{\top} A \xi+1\right) \widehat{\chi_{\varepsilon}}(\xi, s)=\widehat{G \chi_{\varepsilon}}(\xi, s)+\widehat{L \tilde{u}_{\varepsilon}}(\xi, s), \\
(\xi, s) \in \mathbb{R}^{n-1} \times \mathbb{R}^{+}, \\
\widehat{\chi_{\varepsilon}}(\xi, 0)=\widehat{f}(\xi), \quad \xi \in \mathbb{R}^{n-1},
\end{aligned}\right.
$$

where $A=\left\{a_{i j}(0,0)\right\}_{1 \leq i, j \leq n-1}$ is a positive definite symmetric matrix. Notice that

$$
\widehat{\chi_{\varepsilon}}(\xi, s)=0 \quad \forall(\xi, s) \in \mathbb{R}^{n-1} \times[\rho,+\infty) .
$$

To analyze the solution of (3.18) satisfying (3.19), we decompose $\widehat{\chi_{\varepsilon}}(\xi, s)$ as follows. Let

$$
\widehat{\chi_{\varepsilon}}(\xi, s)=w_{\varepsilon}(\xi, s)+v_{\varepsilon}(\xi, s), \quad(\xi, s) \in \mathbb{R}^{n-1} \times \mathbb{R}^{+},
$$


where $w_{\varepsilon}$ satisfies

$$
\left\{\begin{array}{l}
\partial_{s}^{2} w_{\varepsilon}(\xi, s)-\frac{1}{\varepsilon^{2}}\left(\varepsilon^{2} \xi^{\top} A \xi+1\right) w_{\varepsilon}(\xi, s)=0, \quad(\xi, s) \in \mathbb{R}^{n-1} \times \mathbb{R}^{+} \\
w_{\varepsilon}(\xi, 0)=\widehat{f}(\xi), \quad \xi \in \mathbb{R}^{n-1} \\
\lim _{s \rightarrow+\infty} w_{\varepsilon}(\xi, s)=0, \quad \xi \in \mathbb{R}^{n-1}
\end{array}\right.
$$

and $v_{\varepsilon}$ satisfies

$$
\left\{\begin{array}{l}
\partial_{s}^{2} v_{\varepsilon}(\xi, s)-\frac{1}{\varepsilon^{2}}\left(\varepsilon^{2} \xi^{\top} A \xi+1\right) v_{\varepsilon}(\xi, s)=\widehat{G \chi_{\varepsilon}}(\xi, s)+\widehat{L \tilde{u}_{\varepsilon}}(\xi, s), \\
(\xi, s) \in \mathbb{R}^{n-1} \times \mathbb{R}^{+} \\
v_{\varepsilon}(\xi, 0)=0, \quad \xi \in \mathbb{R}^{n-1}, \\
v_{\varepsilon}(\xi, s)=-\widehat{f}(\xi) e^{-s \frac{\sqrt{\varepsilon^{2} \xi^{\top} A \xi+1}}{\varepsilon}}, \quad(\xi, s) \in \mathbb{R}^{n-1} \times[\rho,+\infty) .
\end{array}\right.
$$

The validity of the last equality comes from (3.19) and the following explicit expression of the solution of (3.21):

$$
w_{\varepsilon}(\xi, s)=\widehat{f}(\xi) e^{-s \frac{\sqrt{\varepsilon^{2} \xi^{\top} A \xi+1}}{\varepsilon}} .
$$

We claim that there exists a constant $C>0$ such that for all $\varepsilon \in(0,1)$

$$
\int_{\mathbb{R}^{n-1}}\left|\varepsilon \partial_{s} w_{\varepsilon}(\xi, 0)+w_{\varepsilon}(\xi, 0)\right|^{2} d \xi \leq C \varepsilon^{2}\left\|u_{\varepsilon}\right\|_{H^{1}(\partial \Omega)}^{2} .
$$

Indeed, by (3.23), we get

$$
\begin{aligned}
& \int_{\mathbb{R}^{n-1}}\left|\varepsilon \partial_{s} w_{\varepsilon}(\xi, 0)+w_{\varepsilon}(\xi, 0)\right|^{2} d \xi=\int_{\mathbb{R}^{n-1}}\left(\frac{\varepsilon^{2} \xi^{\top} A \xi}{\sqrt{\varepsilon^{2} \xi^{\top} A \xi+1}+1}\right)^{2}|\widehat{f}(\xi)|^{2} d \xi \\
& \leq \int_{\mathbb{R}^{n-1}} \varepsilon^{2} \xi^{\top} A \xi|\widehat{f}(\xi)|^{2} d \xi
\end{aligned}
$$

and (3.24) follows easily.

Now we need to bound the quantity $\int_{\mathbb{R}^{n-1}}\left|\varepsilon \partial_{s} v_{\varepsilon}(\xi, 0)+v_{\varepsilon}(\xi, 0)\right|^{2} d \xi$ uniformly with respect to $\varepsilon$. This will be done in the next step.

Step 3. Estimate of $\varepsilon \partial_{s} v_{\varepsilon}(\cdot, 0)+v_{\varepsilon}(\cdot, 0)$. We will estimate $\partial_{s} v_{\varepsilon}(\cdot, 0)$ by means of a classical trace theorem. This requires the computation of $\partial_{s}^{2} v_{\varepsilon}$ and $\partial_{s} v_{\varepsilon}$. To do it, we estimate $\widehat{L \widetilde{u}_{\varepsilon}}$ and $\widehat{G \chi_{\varepsilon}}$ first. Throughout the proof, $C$ denotes several positive constants independent of $\varepsilon$.

(a) Estimate of $\widehat{L \widetilde{u}_{\varepsilon}}$ and $\widehat{G \chi_{\varepsilon}}$. Clearly, we have

$$
\left\|\widehat{L \widetilde{u}_{\varepsilon}}\right\|_{L^{2}\left(\mathbb{R}^{n-1} \times \mathbb{R}^{+}\right)} \leq C\left\|u_{\varepsilon}\right\|_{H^{1}(\Omega)} .
$$

By (3.14) and the Plancherel formula, it follows that

$$
\begin{aligned}
\left\|\widehat{G \chi_{\varepsilon}}\right\|_{L^{2}\left(\mathbb{R}^{n-1} \times \mathbb{R}^{+}\right)} & =(2 \pi)^{\frac{n-1}{2}}\left\|G \chi_{\varepsilon}\right\|_{L^{2}\left(\mathbb{R}^{n-1} \times \mathbb{R}^{+}\right)} \\
& \leq(2 \pi)^{\frac{n-1}{2}} \mu_{0} \sum_{i, j=1}^{n-1}\left\|\partial_{h_{i}} \partial_{h_{j}} \chi_{\varepsilon}\right\|_{L^{2}\left(\mathbb{R}^{n-1} \times \mathbb{R}^{+}\right)} \\
& \leq \mu_{0}(n-1)^{2}\left\||\xi|^{2} \widehat{\chi_{\varepsilon}}\right\|_{L^{2}\left(\mathbb{R}^{n-1} \times \mathbb{R}^{+}\right)} .
\end{aligned}
$$


From (3.26) and noting (3.20), we find

$$
\begin{aligned}
\|\widehat{G \chi \varepsilon}\|_{L^{2}\left(\mathbb{R}^{n-1} \times \mathbb{R}^{+}\right)} \leq & \mu_{0}(n-1)^{2}\left\||\xi|^{2} w_{\varepsilon}\right\|_{L^{2}\left(\mathbb{R}^{n-1} \times \mathbb{R}^{+}\right)} \\
& +\mu_{0}(n-1)^{2}\left\||\xi|^{2} v_{\varepsilon}\right\|_{L^{2}\left(\mathbb{R}^{n-1} \times \mathbb{R}^{+}\right)} .
\end{aligned}
$$

On the other hand, multiplying (3.22) by $-|\xi|^{2} \overline{v_{\varepsilon}}$ and then integrating by parts over $\mathbb{R}^{n-1} \times \mathbb{R}^{+}$, taking (3.13) and the last equality of (3.22) into account, we have

$$
\lambda_{0}\left\||\xi|^{2} v_{\varepsilon}\right\|_{L^{2}\left(\mathbb{R}^{n-1} \times \mathbb{R}^{+}\right)} \leq\left\|\widehat{G \chi_{\varepsilon}}\right\|_{L^{2}\left(\mathbb{R}^{n-1} \times \mathbb{R}^{+}\right)}+\left\|\widehat{L \tilde{u}_{\varepsilon}}\right\|_{L^{2}\left(\mathbb{R}^{n-1} \times \mathbb{R}^{+}\right)} .
$$

Substituting (3.28) into (3.27), we get

$$
\begin{aligned}
& \left(1-\frac{\mu_{0}(n-1)^{2}}{\lambda_{0}}\right)\left\|\widehat{G \chi_{\varepsilon}}\right\|_{L^{2}\left(\mathbb{R}^{n-1} \times \mathbb{R}^{+}\right)}+\| \mid \mu_{0}(n-1)^{2} \\
& \leq \mu_{0}(n-1)^{2} w_{\varepsilon}\left\|_{L^{2}\left(\mathbb{R}^{n-1} \times \mathbb{R}^{+}\right)}\right\|_{L^{2}\left(\mathbb{R}^{n-1} \times \mathbb{R}^{+}\right)} .
\end{aligned}
$$

Moreover, from (3.23) and (3.13), we have

$$
\begin{aligned}
\left\||\xi|^{2} w_{\varepsilon}\right\|_{L^{2}\left(\mathbb{R}^{n-1} \times \mathbb{R}^{+}\right)} & =\left(\left.\left.\int_{\mathbb{R}^{n-1}}|| \xi\right|^{2} \widehat{f}(\xi)\right|^{2}\left(\int_{0}^{+\infty} e^{-2 s \frac{\sqrt{\varepsilon^{2} \xi^{\top} A \xi+1}}{\varepsilon}} d s\right) d \xi\right)^{1 / 2} \\
& =\left\|\sqrt{\frac{\varepsilon}{2 \sqrt{\varepsilon^{2} \xi^{\top} A \xi+1}}}|\xi|^{2} \widehat{f}\right\|_{L^{2}\left(\mathbb{R}^{n-1}\right)} \\
& \leq \sqrt{\frac{1}{2 \sqrt{\lambda_{0}}}}\left\||\xi|^{3 / 2} \widehat{f}\right\|_{L^{2}\left(\mathbb{R}^{n-1}\right)} \leq C\left\|u_{\varepsilon}\right\|_{H^{3 / 2}(\partial \Omega) .}
\end{aligned}
$$

Finally, it follows from (3.29), (3.30), and (3.25) that

$$
\|\widehat{G \chi \varepsilon}\|_{L^{2}\left(\mathbb{R}^{n-1} \times \mathbb{R}^{+}\right)} \leq C\left(\left\|u_{\varepsilon}\right\|_{H^{3 / 2}(\partial \Omega)}+\left\|u_{\varepsilon}\right\|_{H^{1}(\Omega)}\right) .
$$

(b) Estimate of $\partial_{s}^{2} v_{\varepsilon}$. Multiplying (3.22) by $\overline{\partial_{s}^{2} v_{\varepsilon}}$ and then integrating by parts over $\mathbb{R}^{n-1} \times \mathbb{R}^{+}$, we obtain, noticing the last equality of (3.22),

$$
\left\|\partial_{s}^{2} v_{\varepsilon}\right\|_{L^{2}\left(\mathbb{R}^{n-1} \times \mathbb{R}^{+}\right)}^{2} \leq\left(\left\|\widehat{G \chi_{\varepsilon}}\right\|_{L^{2}\left(\mathbb{R}^{n-1} \times \mathbb{R}^{+}\right)}+\left\|\widehat{L \tilde{u}_{\varepsilon}}\right\|_{L^{2}\left(\mathbb{R}^{n-1} \times \mathbb{R}^{+}\right)}\right)\left\|\partial_{s}^{2} v_{\varepsilon}\right\|_{L^{2}\left(\mathbb{R}^{n-1} \times \mathbb{R}^{+}\right)} .
$$

This together with (3.25) and (3.31) gives

$$
\left\|\partial_{s}^{2} v_{\varepsilon}\right\|_{L^{2}\left(\mathbb{R}^{n-1} \times \mathbb{R}^{+}\right)} \leq C\left[\left\|u_{\varepsilon}\right\|_{H^{3 / 2}(\partial \Omega)}+\left\|u_{\varepsilon}\right\|_{H^{1}(\Omega)}\right] .
$$

(c) Estimate of $\partial_{s} v_{\varepsilon}$. Noticing the last equality of (3.22), multiplying (3.22) by $-\overline{v_{\varepsilon}}$, and integrating by parts over $\mathbb{R}^{n-1} \times \mathbb{R}^{+}$, we also have

$$
\begin{aligned}
& \left\|\partial_{s} v_{\varepsilon}\right\|_{L^{2}\left(\mathbb{R}^{n-1} \times \mathbb{R}^{+}\right)}^{2}+\frac{1}{\varepsilon^{2}}\left\|v_{\varepsilon}\right\|_{L^{2}\left(\mathbb{R}^{n-1} \times \mathbb{R}^{+}\right)}^{2} \\
& \leq\left\|\varepsilon\left(\widehat{G \chi_{\varepsilon}}+\widehat{L \tilde{u}_{\varepsilon}}\right)\right\|_{L^{2}\left(\mathbb{R}^{n-1} \times \mathbb{R}^{+}\right)}\left\|\frac{v_{\varepsilon}}{\varepsilon}\right\|_{L^{2}\left(\mathbb{R}^{n-1} \times \mathbb{R}^{+}\right)} .
\end{aligned}
$$

Thus,

$$
\left\|\partial_{s} v_{\varepsilon}\right\|_{L^{2}\left(\mathbb{R}^{n-1} \times \mathbb{R}^{+}\right)} \leq \varepsilon\left(\left\|\widehat{G \chi_{\varepsilon}}\right\|_{L^{2}\left(\mathbb{R}^{n-1} \times \mathbb{R}^{+}\right)}+\left\|\widehat{L \widetilde{u}_{\varepsilon}}\right\|_{L^{2}\left(\mathbb{R}^{n-1} \times \mathbb{R}^{+}\right)}\right) .
$$


This together with (3.25) and (3.31) gives

$$
\left\|\partial_{s} v_{\varepsilon}\right\|_{L^{2}\left(\mathbb{R}^{n-1} \times \mathbb{R}^{+}\right)} \leq C\left[\left\|u_{\varepsilon}\right\|_{H^{3 / 2}(\partial \Omega)}+\left\|u_{\varepsilon}\right\|_{H^{1}(\Omega)}\right] .
$$

(d) Estimate of $\partial_{s} v_{\varepsilon}(\cdot, 0)$. We use the following standard inequality:

$$
\begin{aligned}
\int_{\mathbb{R}^{n-1}}\left|\partial_{s} v_{\varepsilon}(\xi, 0)\right|^{2} d \xi & =-2 \int_{\mathbb{R}^{n-1}} \int_{0}^{+\infty} \operatorname{Re}\left(\partial_{s} v_{\varepsilon}(\xi, s) \overline{\partial_{s}^{2} v_{\varepsilon}}(\xi, s)\right) d s d \xi \\
& \leq\left\|\partial_{s} v_{\varepsilon}\right\|_{L^{2}\left(\mathbb{R}^{n-1} \times \mathbb{R}^{+}\right)}^{2}+\left\|\partial_{s}^{2} v_{\varepsilon}\right\|_{L^{2}\left(\mathbb{R}^{n-1} \times \mathbb{R}^{+}\right)}^{2} .
\end{aligned}
$$

This together with (3.32) and (3.33) gives the desired estimate for $v_{\varepsilon}$ :

$$
\int_{\mathbb{R}^{n-1}}\left|\varepsilon \partial_{s} v_{\varepsilon}(\xi, 0)+v_{\varepsilon}(\xi, 0)\right|^{2} d \xi \leq C \varepsilon^{2}\left[\left\|u_{\varepsilon}\right\|_{H^{3 / 2}(\partial \Omega)}^{2}+\left\|u_{\varepsilon}\right\|_{H^{1}(\Omega)}^{2}\right] .
$$

Here we used the fact that $v_{\varepsilon}(\cdot, 0)=0$ given by the second equation of (3.22).

Combining (3.20), the estimates (3.24) and (3.35) imply

$$
\int_{\mathbb{R}^{n-1}}\left|\varepsilon \partial_{s} \widehat{\chi_{\varepsilon}}(\xi, 0)+\widehat{\chi_{\varepsilon}}(\xi, 0)\right|^{2} d \xi \leq C \varepsilon^{2}\left[\left\|u_{\varepsilon}\right\|_{H^{3 / 2}(\partial \Omega)}^{2}+\left\|u_{\varepsilon}\right\|_{H^{1}(\Omega)}^{2}\right],
$$

and hence by the Parseval formula, we obtain

$$
\int_{\mathbb{R}^{n-1}}\left|\varepsilon \partial_{s} \chi_{\varepsilon}(s, 0)+\chi_{\varepsilon}(s, 0)\right|^{2} d s \leq C \varepsilon^{2}\left[\left\|u_{\varepsilon}\right\|_{H^{3 / 2}(\partial \Omega)}^{2}+\left\|u_{\varepsilon}\right\|_{H^{1}(\Omega)}^{2}\right] .
$$

By (3.15), we deduce from (3.37) that

$$
\int_{|s|<\rho / 2}\left|\varepsilon \partial_{s} \tilde{u}_{\varepsilon}(s, 0)+\tilde{u}_{\varepsilon}(s, 0)\right|^{2} d s \leq C \varepsilon^{2}\left[\left\|u_{\varepsilon}\right\|_{H^{3 / 2}(\partial \Omega)}^{2}+\left\|u_{\varepsilon}\right\|_{H^{1}(\Omega)}^{2}\right],
$$

which implies by the change of coordinates involving $\Psi$ that

$$
\int_{\tilde{\Omega}_{x_{0}} \cap \partial \Omega}\left|\varepsilon \frac{\partial u_{\varepsilon}(x)}{\partial \nu}-u_{\varepsilon}(x)\right|^{2} d x \leq C \varepsilon^{2}\left[\left\|u_{\varepsilon}\right\|_{H^{3 / 2}(\partial \Omega)}^{2}+\left\|u_{\varepsilon}\right\|_{H^{1}(\Omega)}^{2}\right]
$$

where $\tilde{\Omega}_{x_{0}} \subset \Omega_{x_{0}}$ is an open neighborhood of $x_{0} \in \partial \Omega$. Since $x_{0}$ is arbitrarily chosen, one easily deduces from (3.39) that

$$
\left\|\varepsilon \frac{\partial u_{\varepsilon}}{\partial \nu}-u_{\varepsilon}\right\|_{L^{2}(\partial \Omega)}^{2} \leq C \varepsilon^{2}\left[\left\|u_{\varepsilon}\right\|_{H^{3 / 2}(\partial \Omega)}^{2}+\left\|u_{\varepsilon}\right\|_{H^{1}(\Omega)}^{2}\right] .
$$

Now, multiplying (3.3) by $u_{\varepsilon}$ and integrating by parts, we find

$$
\left\|\varepsilon \nabla u_{\varepsilon}\right\|_{L^{2}(\Omega)}^{2}+\left\|u_{\varepsilon}\right\|_{L^{2}(\Omega)}^{2}=\varepsilon^{2} \int_{\partial \Omega} \frac{\partial u_{\varepsilon}(x)}{\partial \nu} u_{\varepsilon}(x) d x .
$$

Hence, using the Cauchy-Schwarz inequality, we get

$$
\begin{aligned}
& \varepsilon^{2}\left\|u_{\varepsilon}\right\|_{H^{1}(\Omega)}^{2} \leq \varepsilon\left(\left\|\varepsilon \frac{\partial u_{\varepsilon}}{\partial \nu}-u_{\varepsilon}\right\|_{L^{2}(\partial \Omega)}+\left\|u_{\varepsilon}\right\|_{L^{2}(\partial \Omega)}\right)\left\|u_{\varepsilon}\right\|_{L^{2}(\partial \Omega)} \\
& \leq \frac{\varepsilon}{2 C}\left\|\varepsilon \frac{\partial u_{\varepsilon}}{\partial \nu}-u_{\varepsilon}\right\|_{L^{2}(\partial \Omega)}^{2}+\left(1+\frac{C}{2}\right) \varepsilon\left\|u_{\varepsilon}\right\|_{L^{2}(\partial \Omega)}^{2} .
\end{aligned}
$$


Substituting the above formula into (3.40), we have finally proved that there exists a constant $C>0$ such that for all $\varepsilon \in(0,1)$ any solution $u_{\varepsilon} \in H^{2}(\Omega)$ of

$$
\left(\varepsilon^{2} \Delta-1\right) u_{\varepsilon}(x)=0, \quad x \in \Omega,
$$

satisfies

$$
\left\|\varepsilon \frac{\partial u_{\varepsilon}}{\partial \nu}-u_{\varepsilon}\right\|_{L^{2}(\partial \Omega)}^{2} \leq C \varepsilon\left\|u_{\varepsilon}\right\|_{H^{3 / 2}(\partial \Omega)}^{2} .
$$

Therefore,

$$
\lim _{\varepsilon \rightarrow 0}\left\|\varepsilon \frac{\partial u_{\varepsilon}}{\partial \nu}-u\right\|_{L^{2}\left(\Gamma_{0}\right)}=0
$$

This completes the proof of Theorem 1.2.

Acknowledgments. The special case of Theorem 1.2 in a 2-D disk was first proved in [12] when Bao-Zhu Guo was visiting INRIA in Metz, France in 2002. The authors would like to thank the anonymous referees for their careful reading, helpful suggestions, and many corrections of the manuscript.

\section{REFERENCES}

[1] K. Ammari, Dirichlet boundary stabilization of the wave equation, Asymptot. Anal., 30 (2002), pp. $117-130$.

[2] K. Ammari And M. Tucsnak, Stabilization of second order evolution equations by a class of unbounded feedbacks, ESAIM Control Optim. Calc. Var., 6 (2001), pp. 361-386.

[3] C. I. Byrnes, D. S. Gilliam, V. I. Shubov, and G. Weiss, Regular linear systems governed by a boundary controlled heat equation, J. Dynam. Control Systems, 8 (2002), pp. 341-370.

[4] A. Cheng And K. Morris, Well-posedness of boundary control systems, SIAM J. Control Optim., 42 (2003), pp. 1244-1265.

[5] R. F. Curtain, The Salamon-Weiss class of well-posed infinite dimensional linear systems: A survey, IMA J. Math. Control Inform., 14 (1997), pp. 207-223.

[6] R. F. CURTAIN, Linear operator inequalities for strongly stable weakly regular linear systems, Math. Control Signals Systems, 14 (2001), pp. 299-337.

[7] R. F. Curtain, H. Logemann, and O. J. Staffans, Absolute-stability results in infinite dimensions, Proc. R. Soc. Lond. Ser. A Math. Phys. Eng. Sci., 460 (2004), pp. 2171-2196.

[8] R. F. Curtain and G. Weiss, Well posedness of triples of operators (in the sense of linear systems theory), in Control and Estimation of Distributed Parameter Systems, Internat. Ser. Numer. Math. 91, F. Kappel, K. Kunisch, and W. Schappacher, eds., Birkhäuser, Basel, 1989, pp. 41-59.

[9] R. F. Curtain and H. Zwart, An Introduction to Infinite Dimensional Linear Systems Theory, Springer-Verlag, New York, 1995.

[10] E. A. Guillemin, Synthesis of Passive Networks, Wiley, New York, 1957.

[11] B. Z. Guo AND Y. H. Luo, Controllability and stability of a second order hyperbolic system with colocated sensor/actuator, Systems Control Lett., 46 (2002), pp. 45-65.

[12] B. Z. Guo And C. Z. Xu, Regularity of the Transfer Function of a Wave Equation in a 2-D Disk with Dirichlet Control and Colocated Observation, manuscript, 2002.

[13] B. Z. Guo And G. Q. Xu, Riesz bases and exact controllability of $C_{0}$-groups with onedimensional input operators, Systems Control Lett., 52 (2004), pp. 221-232.

[14] B. JACOB AND H. ZWART, Equivalent conditions for stabilizability of infinite-dimensional systems with admissible control operators, SIAM J. Control Optim., 37 (1999), pp. 1419-1455.

[15] B. JACOB AND H. ZWART, Exact observability of diagonal systems with a finite-dimensional output operator, Systems Control Lett., 43 (2001), pp. 101-109.

[16] B. JaCOB And H. ZWART, Properties of the realization of inner functions, Math. Control Signals Systems, 15 (2002), pp. 356-379.

[17] I. Lasiecka, J. L. Lions, And R. Triggiani, Nonhomogeneous boundary value problems for second order hyperbolic operators, J. Math. Pures Appl. (9), 65 (1986), pp. 149-192. 
[18] J. Lee and G. Uhlmann, Determining anisotropic real-analytic conductives by boundary measurements, Comm. Pure Appl. Math., 42 (1989), pp. 1097-1112.

[19] J. L. Lions, Contrôlabilité exacte, perturbations et stabilisation de systèmes distribués, Tome 1, Contrôlabilité exacte, Recherches en Mathématiques Appliquées 8, Masson, Paris, 1988.

[20] R. Rebarber And G. Weiss, Necessary conditions for exact controllability with a finitedimensional input space, Systems Control Lett., 40 (2000), pp. 217-227.

[21] D. L. Russell and G. Weiss, A general necessary condition for exact observability, SIAM J. Control Optim., 32 (1994), pp. 1-23.

[22] R. Schnaubelt, Feedbacks for nonautonomous regular linear systems, SIAM J. Control Optim., 41 (2002), pp. 1141-1165.

[23] D. Salamon, Infinite dimensional systems with unbounded control and observation: A functional analytic approach, Trans. Amer. Math. Soc., 300 (1987), pp. 383-431.

[24] D. Salamon, Realization theory in Hilbert space, Math. Systems Theory, 21 (1989), pp. 147164.

[25] O. J. Staffans, Quadratic optimal control of well-posed linear systems, SIAM J. Control Optim., 37 (1998), pp. 131-164.

[26] O. J. Staffans, Admissible factorizations of Hankel operators induce well-posed linear systems, Systems Control Lett., 37 (1999), pp. 301-307.

[27] O. J. Staffans, Passive and conservative continuous-time impedance and scattering systems, part I: Well-posed systems, Math. Control Signals Systems, 15 (2002), pp. 291-315.

[28] O. J. Staffans and G. Weiss, Transfer functions of regular linear systems part II: The system operator and the Lax-Phillips semigroup, Trans. Amer. Math. Soc., 354 (2002), pp. 32293262 .

[29] M. TuCSNAK And G. Weiss, How to get a conservative well-posed linear system out of thin air. Part II. Controllability and stability, SIAM J. Control Optim., 42 (2003), pp. 907-935.

[30] G. WeIss, Admissibility of unbounded control operators, SIAM J. Control Optim., 27 (1989), pp. $527-545$.

[31] G. WeIss, Admissibility observation operators for linear semigroups, Israel J. Math., 65 (1989), pp. $17-43$.

[32] G. Weiss, The representation of regular linear systems in Hilbert spaces, in Control and Estimation of Distributed Parameter Systems, F. Kappel, K. Kunisch, and W. Schappacher, eds., Internat. Ser. Numer. Math. 91, Birkhäuser, Basel, 1989, pp. 401-416.

[33] G. WeIss, Regular linear systems with feedback, Math. Control Signals Systems, 7 (1994), pp. 23-57.

[34] G. WeIss, Transfer functions of regular linear systems I: Characterizations of regularity, Trans. Amer. Math. Soc., 342 (1994), pp. 827-854.

[35] G. WeIss, Optimal control of systems with a unitary semigroup and with colocated control and observation, Systems Control Lett., 48 (2003), pp. 329-340.

[36] G. Weiss and R. F. CURTAin, Dynamic stabilization of regular linear systems, IEEE Trans. Automat. Control, 42 (1997), pp. 4-21.

[37] G. Weiss, O. J. Staffans, And M. Tucsnak, Well-posed linear systems-a survey with emphasis on conservative systems, Int. J. Appl. Math. Comput. Sci., 11 (2001), pp. 7-13.

[38] C.-Z. Xu AND G. SAllet, On spectrum and Riesz basis assignment of infinite-dimensional linear systems by bounded linear feedbacks, SIAM J. Control Optim., 34 (1996), pp. 521541 . 